\title{
Establishment and characterization of a novel human colorectal cancer cell line (CLY) metastasizing spontaneously to the liver in nude mice
}

\author{
LIN NA LI ${ }^{1}$, HUA DONG ZHANG ${ }^{2}$, SHOU JUN YUAN $^{1}$, ZENG YUE TIAN $^{1}$ and ZHI XIAN SUN ${ }^{3}$ \\ ${ }^{1}$ Department of Pharmacology and Toxicology, Beijing Institute of Radiation Medicine, 27 Taiping Road, \\ Beijing 100850; ${ }^{2}$ Research Institute of Pharmaceutical Chemistry, P.O.B. 1044, 402 Branch, \\ Beijing 102205; and ${ }^{3}$ Department of Biochemistry and Molecular Biology, Beijing Institute \\ of Radiation Medicine, 27 Taiping Road, Beijing 100850, P.R. China
}

Received October 10, 2006; Accepted November 14, 2006

\begin{abstract}
Colorectal cancer (CRC) with liver metastasis is a fatal disease with rapid progression and poor patient outcome. However, the molecular mechanism involved in liver metastasis of CRC remains essentially unknown, largely because of the presence of few available CRC cell lines with liver metastasis origin and spontaneous metastatic potentials in nude mice. In this study, we established a novel metastatic CRC cell line, CLY, derived from liver metastasis of a 64-yearold Chinese CRC patient. The cell line was characterized by morphology, growth kinetics, tumorigenicity, spontaneous liver metastatic potential, and cytogenetics. Immunofluorescence analysis of $\beta$-catenin and E-cadherin and methylation-specific PCR (MSP) of the E-cadherin gene $(\mathrm{CDH} 1)$ promoter were also used to compare CLY with conventional CRC cell lines (HT-29 and Lovo). CLY exhibited compact and polygonalshaped epithelial cells in vitro and its population doubling time was $29.5 \mathrm{~h}$. Subcutaneous transplantation of CLY into nude mice resulted in subcutaneous tumor formation and spontaneous liver metastasis in all of 10 nude mice. Cytogenetic analysis identified aneuploidy karyotypes with a modal chromosome number of 60. In immunofluorescence analysis, CLY exhibited a low expression but high restricted nuclear localization of $\beta$-catenin and a silenced expression of E-cadherin, which may be induced by hypermethylation of the E-cadherin gene $(\mathrm{CDH})$ promoter and differed from conventional CRC cell lines. Therefore, CLY is an ideal cell model for further exploring the metastatic mechanisms of $\mathrm{CRC}$ and testing new therapeutic reagents for $\mathrm{CRC}$ with liver metastasis.
\end{abstract}

Correspondence to: Professor Shou-Jun Yuan, Department of Pharmacology and Toxicology, Beijing Institute of Radiation Medicine, 27 Taiping Road, Beijing 100850, P.R. China

E-mail: yuansj@nic.bmi.ac.cn

Key words: colorectal cancer, metastasis, cell line, B-catenin, Ecadherin

\section{Introduction}

Colorectal cancer (CRC) is the second most frequent cancer in western countries $(1,2)$. In P.R. China, CRC is the fourth to sixth leading cause of cancer deaths, and its prevalence and mortality are inclined to increase in the coming years (3). Liver metastasis is one of the most important causes of death of CRC patients. In the studies of CRC, some researchers suggest that a large bank of the well-characterized CRC cell lines may provide adequate models for investigating the biological characteristics of CRC. To date, many CRC cell lines are available, but most of them were derived from primary tumors or lymph node metastasis (4-6). CRC cell lines derived from liver metastasis are still few (7). In addition, most CRC cell lines were established from Caucasian patients, with only a few from Chinese patients. Furthermore, there are significant racial differences between Chinese and western CRC patients, such as age, gender, anatomical distribution of primary tumor, CRC stage at diagnosis and survival (8-11). Therefore, it is necessary to provide more CRC cell lines as models to clarify the diversity of CRC and exact mechanisms of liver metastasis. In this study, we established a novel CRC cell line derived from liver metastasis of a Chinese CRC patient and characterized its growth and metastatic potential. The cell line showed a low expression but high restricted nuclear localization of $\beta$ catenin and a silenced expression of E-cadherin in comparison with HT-29 and Lovo cell lines. These results suggested that the CLY cell line is an important model for further exploring the mechanisms of invasion and metastasis of CRC.

\section{Materials and methods}

Primary culture and establishment of cell line. A CRC metastatic tumor specimen from the liver was surgically removed from a 64-year-old Chinese man who was admitted to the 307 hospital (Beijing, P.R. China) and died from liver and lung metastases 6 months after liver partial resection. The fresh tumor tissue specimen was rinsed twice with Hank's balanced salt solution (HBSS), cut into small pieces of $<2 \mathrm{~mm}^{3}$, and then digested with $200 \mathrm{IU} / \mathrm{ml}$ type II collagenase (Worthington Biochemical Corporation, Freehold, NJ, USA) 
in serum-free RPMI-1640 medium (Gibco BRL, Grand Island, $\mathrm{NY}$, USA) for $30-60 \mathrm{~min}$ at $37^{\circ} \mathrm{C}$ in a water bath. After digestion, supernatant fluid was filtered through a $100-\mu \mathrm{m}$ pore mesh and the cells were collected after centrifugation at $1000 \mathrm{r} / \mathrm{min}$. Cell pellets were washed and resuspended in RPMI-1640 medium containing 10\% fetal bovine serum (FBS) (Gibco BRL), $100 \mathrm{U} / \mathrm{ml}$ penicillin and $100 \mu \mathrm{g} / \mathrm{ml}$ streptomycin, and then put into flasks. Cultures were maintained in humidified incubators at $37^{\circ} \mathrm{C}$ in an atmosphere of $5 \% \mathrm{CO}_{2}$ and $95 \%$ air. The medium was replaced twice weekly. After four weeks, growing colonies were harvested with trypsin-EDTA $(0.125 \%)$ $2 \mathrm{mmol} / \mathrm{l}$ ) and seeded in new flasks for passage. Any remaining fibroblasts were removed by mechanical scraping and a differential attachment selection method with trypsin- EDTA, after which pure epithelial cell cultures were obtained. The CLY cell line has now been cultured for over two years and undergone more than 100 passages. The cells of different passage number in fetal bovine serum containing $10 \%$ dimethyl sulfoxide (ACROS, NJ, USA) were frozen and stored in liquid nitrogen during passages.

Growth kinetics in vitro. After establishment, growth kinetics of the CLY cells was obtained by seeding the cells at a concentration of $2 \times 10^{4}$ cells/flask in 40 identical $25-\mathrm{cm}^{2}$ flasks. After seeding, the media were changed every two days. The average viable cell number from three flasks was counted every $24 \mathrm{~h}$ in the presence of trypan-blue dye. The cells were counted for up to 12 days. The growth curve was plotted and the doubling time was calculated during the logarithmic growth phase.

Growth characteristics in vivo. To examine the tumorigenicity and the spontaneous metastatic potential of the CLY cell line in nude mice, logarithmic growing cells were harvested with trypsin-EDTA and $2 \times 10^{6}$ cells in $0.2 \mathrm{ml}$ normal saline were injected subcutaneously into the right abdominal flanks of six-week-old male or female athymic nude mice (BALB/c $n u / n u$, National Institute for the Control of Pharmaceutical and Biological Products, Beijing, P.R. China) maintained under specific-pathogen-free (SPF) conditions. The tumors were measured with a slide caliper every two days and the volumes were estimated by using the following formula: $\mathrm{V}=\mathrm{LxWxH} / 2$ (V=volume; L=length; W=width; H=height). The growth curves of the xenografts were obtained in the 10 nude mice. After 25 days, they were sacrificed for autopsy. Their tumor tissues and livers were removed, fixed in $10 \%$ buffered formalin, and processed for routine histopathologic examination.

Cytogenetic analysis. Eighty-percent confluent monolayer CLY cells were harvested for cytogenetic study. The cells were treated with colcemid (Gibco BRL) at a final concentration of $0.04 \mu \mathrm{g} / \mathrm{ml}$ for $4 \mathrm{~h}$ at $37^{\circ} \mathrm{C}$. The cytogenetic analysis was performed as previously reported (12). The karyotypes of each metaphase were described according to the International System for Human Cytogenetic Nomenclature (13).

Immunofluorescence. CLY and another two CRC cell lines HT-29 and Lovo (HT-29 and Lovo were used as controls. HT-29: human colon adenocarcinoma established from the primary tumor of a 44-year-old Caucasian woman with colon adenocarcinoma in 1964; Lovo: human colon adenocarcinoma established from the metastatic nodule resected from a 56year-old Caucasian man with colon adenocarcinoma in 1972) were seeded on coverslips in growth media $48 \mathrm{~h}$ prior to fixation. The coverslips were washed in PBS and then fixed in ice-cold $100 \%$ acetone for 2 min. After a PBS wash, nonspecific binding was blocked by treatment with $10 \%$ goat serum/PBS for $30 \mathrm{~min}$. Subsequently, the cells were incubated at room temperature for $60 \mathrm{~min}$ with the following primary antibodies: mouse anti-E-cadherin monoclonal antibody and mouse anti- $\beta$-catenin monoclonal antibody (Zymed, San Francisco, CA, USA; diluted 1:70 in blocking solution). After a PBS wash, the cells were then incubated for $45 \mathrm{~min}$ at room temperature with the secondary antibody of fluoroscine isothiocyanate (FITC)-conjugated goat anti-mouse antibody (Zymed; diluted 1:100 in PBS). Then the cells bound with anti- $\beta$-catenin were counterstained with Hoechst 33342 (Sigma, St. Louis, MO, USA) at $5 \mu \mathrm{g} / \mathrm{ml}$ in PBS for another $10 \mathrm{~min}$. After extensive washes with PBS, all the cells were mounted on the slide using $90 \%$ glycerol/PBS. Images were collected on a Bio-Rad Radiance $2100^{\mathrm{TM}}$ confocal system in conjunction with a Nikon TE300 microscope.

Western blot analysis. Logarithmic growing cells (CLY, HT-29 and Lovo) were harvested in ice-cold PBS respectively. Total proteins of each cell line were extracted and separated by SDS-PAGE and transferred onto polyvinylidene difluoride membrane, as described previously $(14,15)$. The membrane was blocked for $2 \mathrm{~h}$ at room temperature in Tris-buffered saline containing $0.1 \%$ Tween-20 and 5\% dried skimmed milk powder. The membrane was probed with primary antibodies against $\beta$-catenin and $\beta$-actin (Santa Cruz, CA, USA) at a dilution of 1:300 in blocking solution for $2 \mathrm{~h}$ at room temperature. After serial washes with Tris-buffered saline containing $0.1 \%$ Tween-20, the membrane was incubated with the horseradish peroxidase-conjugated secondary antibody (Santa Cruz) at a dilution of 1:400. Immunoreactive proteins were detected by using an enhanced chemiluminescence system (Santa Cruz).

Methylation-specific PCR (MSP) for the E-cadherin gene $(C D H 1)$ promoter. Promoter methylation was studied in CDH1 by MSP, a method that distinguishes unmethylated from methylated alleles of a given gene (16). After bisulphite treatment of DNA, which converts unmethylated but not methylated cytosines to uracil, DNA is amplified by PCR using primers specific to methylated and unmethylated sequences.

Genomic DNAs were extracted from CLY and the other two CRC cell lines HT-29 and Lovo using a genomic DNA extracting kit (Tiangen Biotech, Beijing, P.R. China), respectively. One $\mu \mathrm{g}$ DNA from each cell line was modified as described (17). Previously reported primer sets and PCR conditions were used for amplification of the $C D H 1$ fragments $(18,19)$. The PCR products were loaded onto $2 \%$ agarose gels containing ethidium bromide, and visualized by UV illumination after electrophoresis. Lind et al (19) reported that $\mathrm{CDH1}$ promoters of HT-29 and Lovo were unmethylated, so the two CRC cell lines can be used as controls. 
A

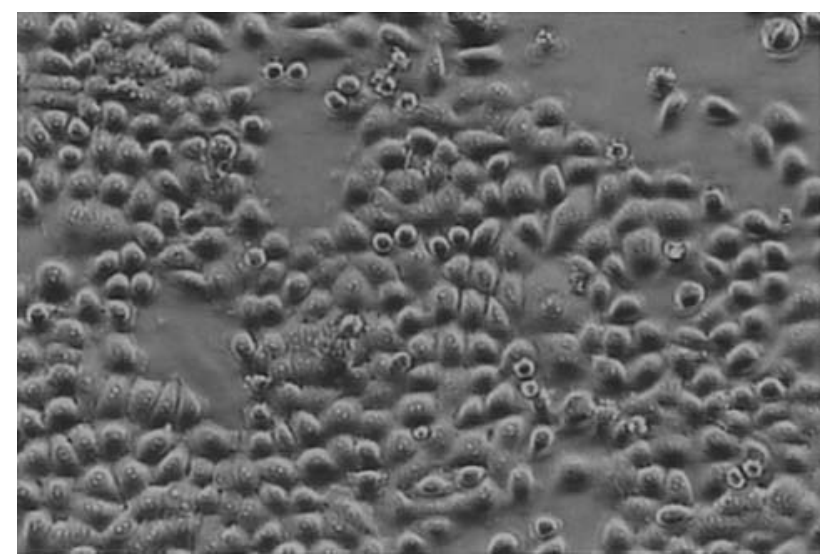

B

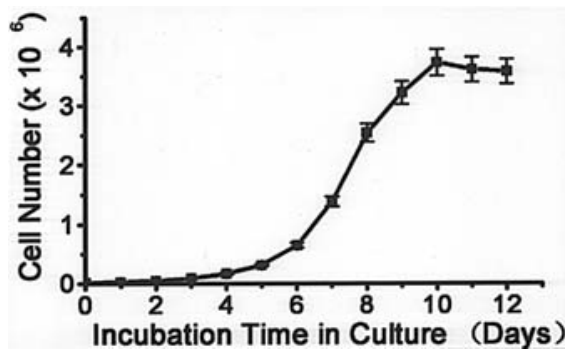

Figure 1. Morphology and growth kinetics of CLY cells in vitro. (A) Phasecontrast micrograph of CLY demonstrating prominent adherent epithelioid cells (original magnification, $x 400$ ). (B) Growth curve of CLY cells in RPMI-1640 supplemented with $10 \%$ fetal bovine serum. The in vitro population doubling time was $29.5 \mathrm{~h}$.

\section{Results}

Establishment of CLY cell line. Twenty-four hours after the primary culture, some colonies of epithelial-like cells and fibroblasts attached to culture flasks. The colonies were inactive and stable for about 3 weeks while fibroblasts grew fast. Contaminating fibroblasts were removed by scraping and differential trypsinization. One month after the cell culture initiation, the epithelial-like cells became active and propagated as monolayer cells. After 12 successive passages, a pure tumor cell line with continuous and stabilized multiplication was established and designated as CLY. The cell line has grown continuously over 100 passages during the past two years without apparent phenotypic change. The stored cells were also propagated in culture without a noticeable change in morphology after recovery from cryopreservation.

Morphology of CLY. Under a phase-contrast microscope, CLY exhibited features of epithelial cells with polygonal shape and adherent growth in a monolayer (Fig. 1A).

Growth kinetics in vitro. CLY followed a typical growth curve, including lag, logarithmic and stationary phases during the 12-day culture (Fig. 1B). The population doubling time in logarithmic growth phase was approximately $29.5 \mathrm{~h}$.

Growth characteristics in vivo. CLY showed high tumorigenicity and spontaneous metastatic potential in athymic
A

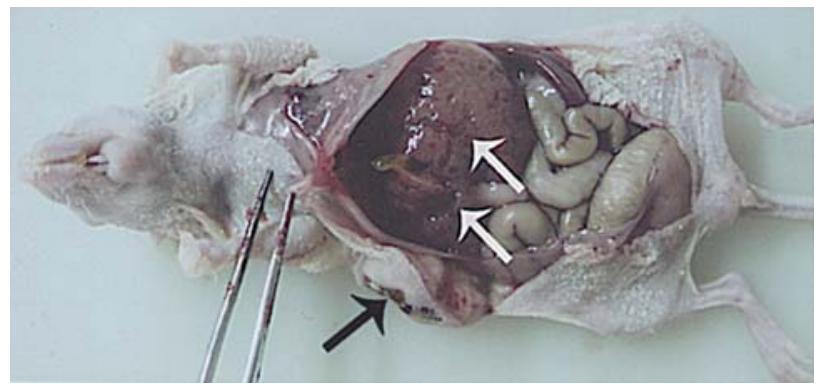

B

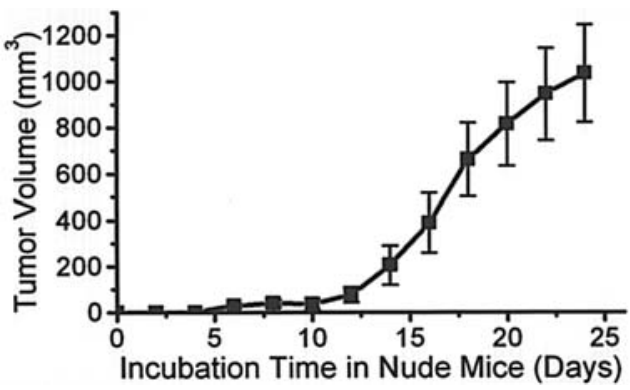

C

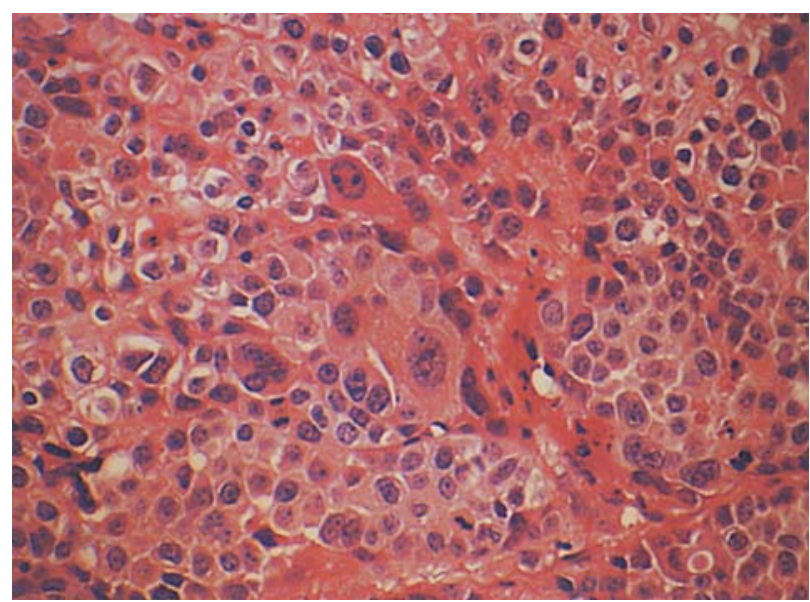

D

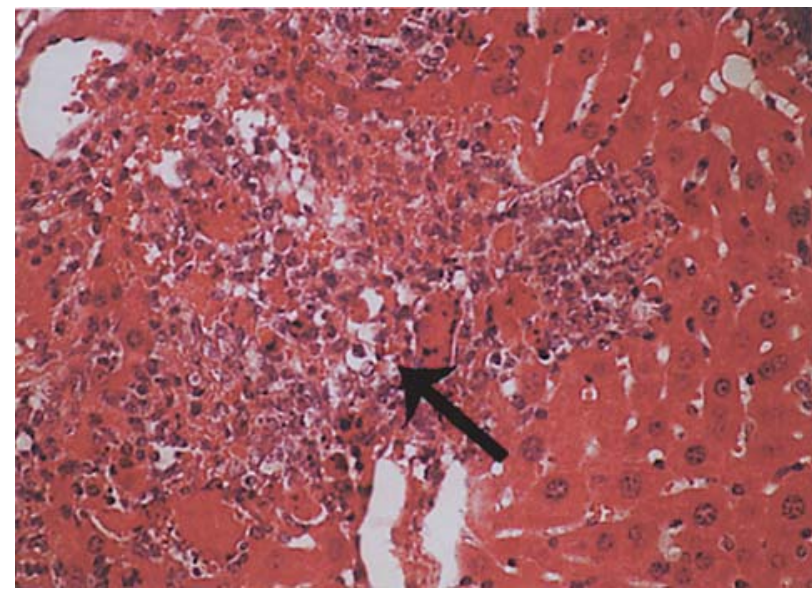

Figure 2. Tumorigenicity, growth characteristics and spontaneous metastatic potentials of CLY cells in nude mice. (A) Primary tumor (black arrow) and liver metastases (white arrows) formed 25 days after subcutaneous transplantation of CLY cells in nude mice. (B) Growth curve of subcutaneous tumors. The tumor doubling time during the exponential phase of growth was $71.3 \mathrm{~h}$. (C) Histopathology of subcutaneous tumors. (hematoxylin and eosin, original magnification, $\mathrm{x} 400$ ). (D) Photomicrographs of liver metastases (black arrow) (hematoxylin and eosin, original magnification, x200). 


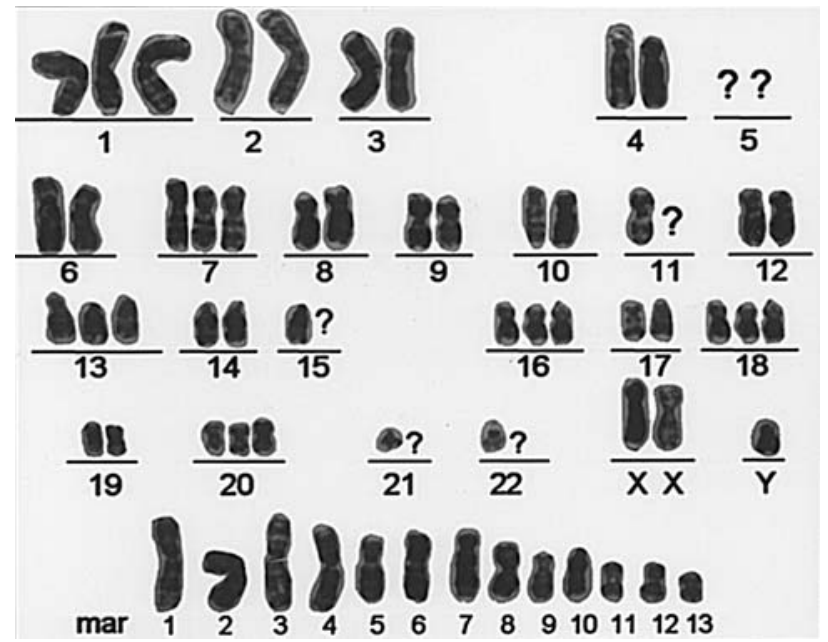

Figure 3. Representative G-banded karyotype of CLY showing chromosomal abnormalities and marker chromosome (mar): $60,+\mathrm{XXY},+1,-5,-5,+7,-11$, $+13,-15,+16,+18,+20,-21,-22,+$ mar.

A
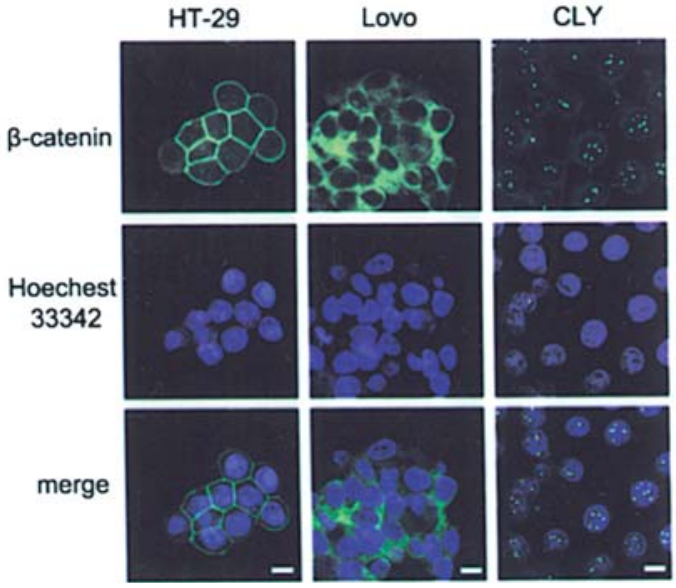

B

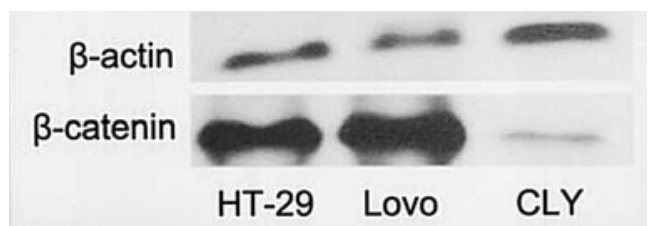

Figure 4. Subcellular distribution and expression levels of $ß$-catenin in three CRC cell lines, HT-29 (as a control), Lovo (as a control), and CLY. (A) Subcellular distribution of $ß$-catenin (ß-catenin labeling, green; and nuclear stained by Hoechst 33342 labeling, blue). Scale bars $=10 \mu \mathrm{m}$. (B) Western blot analysis for $\beta$-catenin. $\beta$-actin was used as the loading control.

nude mice. Within 5-7 days after subcutaneous injection of the cells, visible subcutaneous tumors developed in all of the 10 nude mice at the sites of inoculation. After 25 days, the tumors grew up to about $1000 \mathrm{~mm}^{3}$ (Fig. 2B). During the logarithmic phase of growth, the doubling time of the tumors was $71.3 \mathrm{~h}$. Macroscopically, the tumors were well encapsulated and attached to the covering dermas (Fig. 2A). Microscopically, the tumors were classified as poorly differentiated tubular adenocarcinoma (Fig. 2C). Metastases to liver occurred in all the mice (Fig. 2A and D).
A

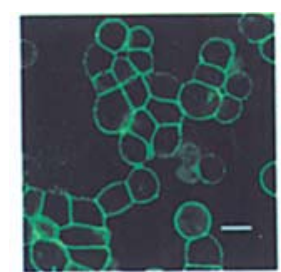

HT-29

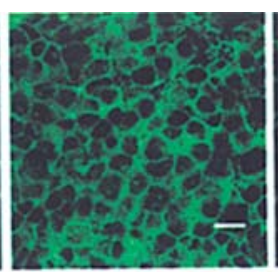

Lovo

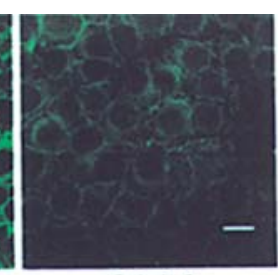

CLY
$\mathbf{B}$

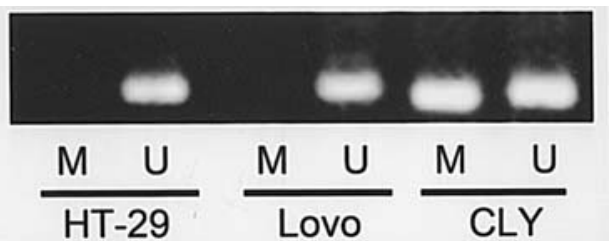

Figure 5. Expression of E-cadherin and methylation status of E-cadherin gene (CDH1) promoter in three CRC cell lines HT-29 (as a control), Lovo (as a control), and CLY. (A) Immunoreactivity of E-cadherin. Scale bars $=10 \mu \mathrm{m}$. (B) The results of MSP of the E-cadherin gene (CDH1). M, methylation specific PCR product; $U$, unmethylation specific PCR product.

Chromosome analysis. A cytogenetics study revealed the number of chromosomes ranging between 56 and 92 with a modal chromosome number of 60 . The G-banded analysis demonstrated aneuploidy karyotype and several marker chromosomes (Fig. 3). Therefore, CLY is a CIN (chromosomal instability, i.e. aneusomy, gains and losses of chromosomal regions) cancer cell line.

Subcellular distribution and expression levels of $\beta$-catenin. $\beta$-catenin had a definite nuclear localization in CLY. In comparison, there was a dispersed distribution of B-catenin in cytoplasm of Lovo and a highly restricted distribution in plasma membrane of HT-29, especially at the cell junction (Fig. 4A).

Western blot analysis also revealed that the expression level of $\beta$-catenin was much lower in CLY than in HT-29 and Lovo (Fig. 4B).

Expression of E-cadherin and hypermethylation of E-cadherin gene (CDH1) promoter. E-cadherin was strongly labeled in the plasma membrane of HT-29, and also present in the plasma membrane and cytoplasm of Lovo. In contrast, the immunoreactivity of E-cadherin was largely reduced in CLY (Fig. 5A).

A clearly visible band in lane M of CLY demonstrated hypermethylation of its $\mathrm{CDH} 1$ promoter. No amplification products were produced with methylated primers (lanes $\mathrm{M}$ of HT-29 and Lovo), while the unmethylated primers amplified clearly visible bands (lanes U of HT-29 and Lovo), which indicated that $\mathrm{CDH} 1$ promoters of HT-29 and Lovo were unmethylated (Fig. 5B).

\section{Discussion}

Well-characterized human cancer cell lines are important research resources for studying cancer cell biology, as well as for developing new strategies against cancer cell growth, progression, and metastasis. 
In this study, we reported the establishment and characterization of a new CRC cell line (CLY), derived from liver metastasis of a Chinese CRC patient. In vitro, CLY grew as an adherent monolayer with characteristic epithelial morphology. The cultured cells maintained consistent morphology during the following subculture passages. In vivo, CLY showed high tumorigenicity in athymic nude mice and the xenotransplanted tumors were classified as poorly differentiated tubular adenocarcinoma that was similar to pathological diagnosis of the Chinese CRC patient. In addition, cytogenetic analysis revealed that CLY was a CIN carcinomous cell line which occurred in approximately $85 \%$ of colorectal tumors (20). These results demonstrated the cell line shared the common biological features of CRC tumors.

One of the most noticeable characteristics of CLY was its highly spontaneous metastatic potential to the liver in nude mice. Liver metastasis is one of the major routes for metastasis of CRC. The metastatic cascade starts with a breakdown of the epithelial integrity which enables tumour cells to leave epithelial structures, to invade the surrounding stroma, to enter either blood or lymphatic vessels and extravasate in the appropriate target organs. Epithelial cadherin (E-cadherin) is an intercellular adhesion molecule of epithelial cells, known to play an important role in maintaining the morphological integrity of normal mucosa in the presence of $\mathrm{Ca}^{2+}(21)$. Decreased membranous E-cadherin expression may free cancer cells from their adhesive constrain, allowing them to migrate out of their primary tumors (22). In the cytoplasm, the intracellular domain of E-cadherin is being linked to the contractile cytoskeleton by a complex of different proteins: $\alpha$ - , $\beta$-catenin, and $\gamma$-catenin (21). Of particular importance is B-catenin, which not only plays an essential role in cell-cell adhesion, but also acts as a central player in Wnt/B-catenin pathway (23-26). Reduced expression of E-cadherin also leads to $\beta$-catenin disassociating from adherent complex and transferring to cytoplasm. Instead of degrading in cytoplasm, B-catenin was translocated into the nucleus, where it may subsequently bind with transcription factors TCF/LEF to activate transcription of target genes that have important roles in the development and progression of colorectal cancer, namely: $c-m y c$, cyclin D1, gastrin, COX-2, MMP-7, uPAR, CD44 and P-glycoprotein (27).

In CLY cells, hypermethylation of the E-cadherin gene $(\mathrm{CDH1})$ promoter may induce reduced expression of $\mathrm{E}$ cadherin. The low expression of E-cadherin not only may free cancer cells from their adhesive constraint, but also may lead to nuclear translocation of $B$-catenin that is often associated with progression and metastasis of CRC (28-30). Thus the low expression of E-cadherin and the highly restricted nuclear localization of B-catenin may explain its potent aggressive and metastatic nature in nude mice.

Some researchers also refer to metastatic cancer cells as 'migrating cancer stem (MCS) cells' (31). In colorectal cancer they characterised potential MCS cells as the cells that express high levels of nuclear $\beta$-catenin (31). Nuclear $\beta$-catenin is involved in two fundamental processes in embryonic development: epithelial-mesenchymal transition (EMT) and stem cell formation. Accumulating data demonstrate that aberrant nuclear expression of $\beta$-catenin can also confer these two abilities to tumor cells, thereby driving malignant tumor progression
(32). According to these opinions, CLY may possess some stem-cell-like characteristics and be worthy of further study.

In conclusion, we established and characterized a novel metastatic human CRC cell line (CLY) with Chinese origin and highly spontaneous metastatic potentials. Particularly, the cell line was observed to have a low expression of E-cadherin and a highly restricted nuclear localization of $\beta$-catenin. These characteristics indicate that the CLY cell line can provide us with a new experimental system to study the pathogenic mechanism, to investigate the biological behavior, and to evaluate new therapeutic agents for CRC with liver metastasis.

\section{Acknowledgements}

We thank Professor Jian-Ming Xu for providing tumor specimens and Ms Chuan-Hua Zhao for technical assistance.

\section{References}

1. Steinert R, Buschmann T, van der Linden M, Fels LM, Lippert H and Reymond MA: The role of proteomics in the diagnosis and outcome prediction in colorectal cancer. Technol Cancer Res Treat 1: 297-304, 2002.

2. Price AS: Primary and secondary prevention of colorectal cancer. Gastroenterol Nurs 26: 73-81, 2003.

3. Zhang YZ and Li SY: New technologies of early diagnosis on colorectal cancer. Shijie Huaren Xiaohua Zazhi 12: 1202-1206, 2004.

4. Data from the American Type Culture Collection (ATCC): http://www .atcc.org/ documents/pdf/tcl.pdf

5. Oh JH, Ku JL, Yoon KA, et al: Establishment and characterization of 12 human colorectal-carcinoma cell lines. Int J Cancer 81: 902-910, 1999.

6. McBain JA, Weese JL, Meisner LF, Wolberg WH and Willson JK: Establishment and characterization of human colorectal cancer cell lines. Cancer Res 44: 5813-5821, 1984.

7. Yamachika T, Nakanishi H, Yasui K, et al: Establishment and characterization of a human colonic mucinous carcinoma cell line with predominant goblet-cell differentiation from liver metastasis. Pathol Int 55: 550-557, 2005.

8. Chien C, Morimoto LM, Tom J and Li CI: Differences in colorectal carcinoma stage and survival by race and ethnicity. Cancer 104: 629-639, 2005.

9. Lieberman D: Race, gender, and colorectal cancer screening. Am J Gastroenterol 100: 2756-2758, 2005.

10. Soon MS, Kozarek RA, Ayub K, Soon A, Lin TY and Lin OS: Screening colonoscopy in Chinese and Western patients: a comparative study. Am J Gastroenterol 100: 2749-2755, 2005.

11. Qing SH, Rao KY, Jiang HY and Wexner SD: Racial differences in the anatomical distribution of colorectal cancer: a study of differences between American and Chinese patients. World J Gastroenterol 9: 721-725, 2003.

12. Wan TS, Chan LC, Ngan HY and Tsao SW: t(High) frequency of telomeric associations in human ovarian surface epithelial cells transformed by human papilloma viral oncogenes. Cancer Genet Cytogene 95: 166-172, 1997.

13. ISCN: An International System for Human Cytogenetic Nomenclature. F Mitelman (ed). S. Karger, Basel, 1995.

14. Gardner SH, Hawcroft G and Hull MA: Effect of nonsteroidal anti-inflammatory drugs on beta-catenin protein levels and catenin-related transcription in human colorectal cancer cells. Br J Cancer 91: 153-163, 2004.

15. Mariner DJ, Davis MA and Reynolds AB: EGFR signaling to p120-catenin through phosphorylation at Y228. J Cell Sci 117: 1339-1350, 2004.

16. Herman JG, Graff JR, Myohanen S, Nelkin BD and Baylin SB: Methylation-specific PCR: a novel PCR assay for methylation status of CpG islands. Proc Natl Acad Sci USA 93: 9821-9826, 1996.

17. Smith-Sorensen B, Lind GE, Skotheim RI, et al: Frequent promoter hypermethylation of the O6-Methylguanine-DNA Methyltransferase (MGMT) gene in testicular cancer. Oncogene 21: 8878-8884, 2002. 
18. Graff JR, Herman JG, Myohanen S, Baylin SB and Vertino PM: Mapping patterns of $\mathrm{CpG}$ island methylation in normal and neoplastic cells implicates both upstream and downstream regions in de novo methylation. J Biol Chem 272: 22322-22329, 1997.

19. Lind GE, Thorstensen L, Lovig T, et al: A CpG island hypermethylation profile of primary colorectal carcinomas and colon cancer cell lines. Mol Cancer 3: 28-38, 2004.

20. Grady WM: Genomic instability and colon cancer. Cancer Metastasis Rev 23: 11-27, 2004.

21. Kemler R and Ozawa M: Uvomorulin-catenin complex: cytoplasmic anchorage of a $\mathrm{Ca}^{2+}$-dependent cell adhesion molecule. Bioessays 11: 88-91, 1989.

22. Kitagawa T, Matsumoto K, Nagafuchi A, Tsukita S and Suzuki H: Co-expression of E-cadherin and alpha-catenin molecules in colorectal cancer. Surg Today 29: 511-518, 1999.

23. Nelson WJ and Nusse R: Convergence of Wnt, beta-catenin, and cadherin pathways. Science 303: 1483-1487, 2004.

24. Bienz M: beta-Catenin: a pivot between cell adhesion and Wnt signalling. Curr Biol 15: R64-R67, 2005.

25. Harris TJ and Peifer M: Decisions, decisions: beta-catenin chooses between adhesion and transcription. Trends Cell Biol 15: 234-237, 2005.
26. Brembeck FH, Rosario M and Birchmeier W: Balancing cell adhesion and Wnt signaling, the key role of beta-catenin. Curr Opin Genet Dev 16: 51-59, 2006.

27. Wong NA and Pignatelli M: Beta-catenin - a linchpin in colorectal carcinogenesis? Am J Pathol 160: 389-401, 2002.

28. Khoursheed MA, Mathew TC, Makar RR, et al: Expression of E-cadherin in human colorectal cancer. Surgeon 1: 86-91, 2003.

29. Wong SC, Lo ES, Lee KC, Chan JK and Hsiao WL: Prognostic and diagnostic significance of beta-catenin nuclear immunostaining in colorectal cancer. Clin Cancer Res 10: 1401-1408, 2004.

30. Kojima M, Shiokawa A, Ohike N, Tamegai Y, Kato H and Morohoshi T: Relation of E-cadherin and beta-catenin expression to pit pattern in colorectal cancer. Oncol Rep 12: 533-538, 2004.

31. Brabletz T, Jung A, Spaderna S, Hlubek F and Kirchner T: Opinion: migrating cancer stem cells - an integrated concept of malignant tumour progression. Nat Rev Cancer 5: 744-749, 2005.

32. Brabletz T, Hlubek F, Spaderna S, Schmalhofer O, Hiendlmeyer E, Jung A and Kirchner T: Invasion and metastasis in colorectal cancer: epithelial-mesenchymal transition, mesenchymal-epithelial transition, stem cells and beta-catenin. Cells Tissues Organs 179: 56-65, 2005. 